\title{
Enfermedad de Mondor y mamoplastías
}

\author{
Jorge Osvaldo Jury S. ${ }^{1}$, Catalina Ignacia Jury S. ${ }^{2}$, \\ Brian Adams Morales O. ${ }^{2}$ y Osvaldo Andrés Jury S. ${ }^{3}$
}

\section{Mondor`s disease and mammoplasty}

Aim: Review the disease incidence in patients who had mammoplasties in a private clinic on a 11 yearperiod. The purpose is to describe their symptoms and signs, diagnosis, treatment and evolution. Materials and Method: 355 women who underwent cosmetic mammoplasties were studied. Those reconstructive surgeries secondary to breast cancer and those with other previous oncological history were excluded. The information was collected from clinical files. Results: 710 breasts operated between 2008-2018 are analyzed, six of them (6/710) evolved Mondor disease (0.8\%). The diagnosis was made clinically. All cases received oral and local anti-inflammatory therapy, responding favorably. Discussion: There is a few published literature about it. Bilateral cases are less reported. It appears to be associated with surgeries of the submammary sulcus and it is not clear that it requires therapeutic cure. Conclusions: Mondor's disease is infrequent, can occur secondary to mammoplasty, diagnosis is clinical, treatment is symptomatic, all cases had satisfactory evolution without sequelae.

Key words: Mondor; thrombophlebitis; breasts.

\section{Resumen}

Objetivo: Revisar la incidencia de esta enfermedad en pacientes sometidos a mamoplastías en una clínica privada en el período de 11 años, con el fin de describir sus síntomas y signos, diagnóstico, tratamiento y evolución. Materiales y Método: Se estudiaron 355 mujeres que se sometieron a mamoplastías estéticas. Se excluyó aquellas cirugías reconstructivas secundarias a cáncer de mama y aquellas con otros antecedentes oncológicos previos. La información fue recolectada de las fichas clínicas. Resultado: Se analizan 710 mamas operadas en el período 2008-2018, de las cuales seis mamas (6/710) evolucionaron con enfermedad de Mondor $(0,8 \%)$. El diagnóstico se realizó clínicamente. Todos los casos recibieron terapia antiinflamatoria oral y local, respondiendo favorablemente. Discusión: Existe poca literatura publicada al respecto. Casos bilaterales son menos reportados. Pareciera asociarse con cirugías del surco submamario y no está claro que requiera curación terapéutica. Conclusiones: La enfermedad de Mondor es infrecuente, puede presentarse secundario a mamoplastías, es de diagnóstico clínico y sólo tratamiento sintomático con evolución satisfactoria sin secuelas.

Palabras clave: Mondor; tromboflebitis; mamas.
Cirujano Plástico, Clínica Sanatorio Alemán de Concepción. Concepción, Chile.

IInternos de Medicina, Facultad de Medicina, Universidad Católica de la Santísima Concepción. Concepción, Chile. ${ }^{3}$ Alumno de Medicina, Facultad de Medicina, Universidad San Sebastián. Concepción, Chile.

Recibido 2020-03-16 y aceptado 2020-05-14

Correspondencia a: Dr. Jorge Jury S. juryplastic@gmail.com

\section{Introducción}

La enfermedad de Mondor o tromboflebitis superficial de la pared anterolateral del tórax ${ }^{1}$ fue descrita por primera vez por Henry Mondor, cirujano francés, en $1939^{2}$. Es una enfermedad poco prevalente cuya etiología parece estar relacionada con tumores, traumatismos y cirugías que afectan con mayor frecuencia a las venas toracoepigástricas, torácicas laterales y epigástricas superiores, que dre- nan las áreas lateral e inferior de las $\operatorname{mamas}^{3}$ (Figura 1), habitualmente ocurre en pacientes de mediana edad del género femenino ${ }^{4}$, se ha descrito en otras áreas como en brazos y pene ${ }^{5}$.

El objetivo de esta revisión es identificar la incidencia de la enfermedad de Mondor secundaria a mamoplastías, en el período comprendido entre 2008-2018, en una clínica privada con el fin de describir sus síntomas, signos, diagnóstico, tratamiento y evolución. 


\section{Materiales y Método}

Se presenta un estudio observacional de una serie de casos de 355 mujeres que recibieron mamoplastía. El estudio fue comprendido entre enero de 2008 y diciembre de 2018 (once años) en la clínica Sanatorio Alemán de Concepción, Chile. Se excluyeron todas aquellas pacientes con mamoplastías reconstructivas secundarias a cáncer de mamas $\mathrm{u}$ de otro origen y aquellas con factores de riesgo o antecedente de trombosis venosa superficial o profunda. Todas fueron estudiadas previamente con imagenología mamaria. La información fue recolectada de las fichas clínicas personales. De los 355 casos, 201 correspondieron a mamoplastías de aumento bilateral con implantes (402 mamas), 135

Figura 1. Anatomía venas toráco-epigástricas, torácicas laterales y epigástricas superiores.

Figura 2. Paciente con mamoplastía de aumento con implantes. Mondor bilateral. a mamoplastías reductivas bilateral (270 mamas reducidas) y 19 casos con asimetría en que se realizó combinación de aumento en un lado (19 mamas) y reducción contralateral (19 mamas). El universo corresponde a 355 pacientes o 710 mamas, de las cuales 421 recibieron implantes y 289 reducción.

Las variables a estudiar fueron edad, tiempo de inicio de síntomas, duración de la enfermedad, lateralidad, tipo de cirugía (aumento o reducción), tratamiento y evolución.

Se realizó estadística descriptiva en el manejo de las variables.

\section{Resultados}

Esta serie está compuesta por 355 mujeres operadas, que presentan una mediana de edad de 44 años. 201/355 (57\%) recibieron mamoplastías de aumento, 135/355 (38\%) mamoplastías reductivas y 19/355 (5\%) que recibieron asociación de reducción en un lado y aumento en el otro por presentar asimetría mamaria, lo que determina un universo de 710 mamas. De ellas, 421/710 (59\%) recibieron implantes y 289/710 (41\%) recibieron reducción. La incidencia de enfermedad de Mondor fue de 5/355 $(1,4 \%)$ del total de pacientes y $6 / 710(0,8 \%)$ del total de las mamas operadas. De las mamas implantadas se presentó enfermedad de Mondor en 4/421 $(0,95 \%)$ y entre las reducidas fue de $2 / 289(0,7 \%)$.

De las pacientes con Mondor hubo 1/5 (20\%) que presentó bilateralidad (Figura 2) y correspondía a cirugía de aumento. Las otras 4 pacientes, presentaron unilateralidad, $2 / 5$ (40\%) correspondieron a cirugía reductiva bilateral y $2 / 5(40 \%)$ a mamoplastía de aumento con implantes.

En todos los casos, el abordaje quirúrgico involucró el surco submamario. La clínica de presentación de la enfermedad de Mondor fue dolor y presencia de cordón irregular en la pared torácica. El promedio del inicio de síntomas correspondió a los 11 días poscirugía, siendo 7 días el tiempo mínimo de aparición y 15 días el máximo.

En todos los casos la tromboflebitis se presentó distal a los cuadrantes inferiores de las mamas haciéndose más evidente con los brazos elevados.

Todos los casos fueron tratados para aliviar su sintomatología con antiinflamatorios no esteroidales, ketoprofeno (Profenid B, Sanofi-Aventis, Chile S.A.) $150 \mathrm{mg}$ oral cada $12 \mathrm{~h}$ por 7 días y con Arnika gel 10\% (Arnikaderm gel 10\% Laboratorio Knop, Chile S.A.) tópico cada $12 \mathrm{~h}$ por 7 días. En dos casos se complementó con ultrasonido diario por 10 sesiones, no existiendo criterios de selec- 
ción para este último, sin embargo, no se registró diferencias en la duración de los signos ni síntomas entre ellas.

Los 6 casos se resolvieron, desapareciendo su signología en un máximo de 10 semanas. No hubo secuelas ni recidivas, en el seguimiento a un año.

\section{Discusión}

Nuestros hallazgos son similares a los descritos en la literatura nacional e internacional ${ }^{6,7}$, coincidiendo en que la enfermedad de Mondor puede ser secundario a mamoplastias ${ }^{5}$. Cuando se asocia a traumatismos o cirugía, algunas publicaciones describen que estaría en relación al daño de la capa íntima de venas ${ }^{3}$. En esta serie todas las pacientes tuvieron incisión a nivel del surco submamario, lo que se menciona como probable causa de esta enfermedad ${ }^{7}$. Otras etiologías descritas, son neoplasias y enfermedades reumatológicas como artritis reumatoide $^{8}$ y vasculitis sistémica ${ }^{9}$, probablemente por estados de hipercoagulabilidad. Una publicación señala como causal movimientos repetitivos y excesivos de brazos $^{8}$. No existe unanimidad de causalidad en la literatura publicada.

El diagnóstico es fundamentalmente clínico, sobre todo si existe el antecedente de cirugía reciente, sin embargo, se ha descrito que la ecografía Doppler ayuda al diagnóstico ${ }^{6}$. En esta serie fue suficiente la manifestación clínica.

No existe claridad ni uniformidad sobre el tratamiento. La mayoría de las publicaciones coinciden en el uso de analgesia y antiinflamatorios mientras ocurre la resolución espontánea de esta enfermedad, que se estima en promedio 8 semanas $^{7}$. Si bien, no se menciona la anticoagulación terapéutica en los artículos revisados sobre enfermedad de Mondor, en aquellos que tratan sobre tromboflebitis superficial de extremidades inferiores, recomiendan diversos esquemas en casos más severos ${ }^{10}$, sin embargo, dadas las características autolimitadas en esta enfermedad, podría constituir un sobretratamiento. También se menciona el uso de acetónido de triamcinolona ${ }^{11}$, intralesional en la dermis del cordón subcutáneo.

La evolución natural de esta enfermedad es hacia la resolución espontánea. La terapia puede contribuir a disminuir los síntomas ${ }^{12}$.

\section{Conclusiones}

La enfermedad de Mondor es poco frecuente y puede ocurrir secundaria a mamoplastías. Hay pocas publicaciones sobre casos bilaterales. Su diagnóstico es clínico y el tratamiento principalmente sintomático. Su evolución es hacia la recuperación espontánea, sin secuelas.

\section{Responsabilidades éticas}

Protección de personas y animales. Los autores declaran que para esta investigación no se han realizado experimentos en seres humanos ni en animales.

Confidencialidad de los datos. Los autores declaran que en este artículo no aparecen datos de pacientes. Además, se cuenta con consentimiento escrito de paciente para mostrar imágenes.

Conflictos de interés: no hay.

\section{Bibliografía}

1. Herrán MFS, Lugo BI, Madrid RA, Baquero UM. Enfermedad de Mondor de la mama. Reporte de caso bilateral. Cir Plast. 2013;23:153-7.

2. Laroche JP, Galanaud J, Labau D, Van Kien AK, Brisot D, Boge G, et al. Mondor's disease: what's new since 1939 ? Thromb Res. 2012;130:S56-8.

3. Loos B, Horch RE. Mondor's Disease after Breast Reduction Surgery. Plastic and Reconstructive Surgery 2006;117:129e-32e.

4. Villalobos C, Mora G. Actualización en enfermedad de Mondor. Medicina Legal de Costa Rica 2017;34:244-9.
5. Krause S, Lüdecke G, Weidner W. Mondor's disease of the penis. Urol Int. 2000;64:99-100.

6. Álvarez-Garrido H, Garrido-Ríos A, Sanz-Muñoz C, Miranda-Romero A. Mondor's disease. Clin Exp Dermatol. 2009;34:753-6.

7. Khan UD. Incidence of Mondor Disease in Breast Augmentation: A Retrospective Study of 2052 Breasts Using Inframammary Incision. Plastic and Reconstructive Surgery 2008;122:88e-9e.

8. Díaz-Miguela V, Gonzalo I, Tello A, Alonso E. Tromboflebitis superficial de la pared torácica: enfermedad de Mondor idiopática. Clínica e investigación en ginecología y obstetricia 2006;33;64-6. DOI: 10.1016/S0210-573X(06)74086-2

9. Rountree KM, Barazi H, Aulick NF. Mondor Disease. In: StatPearls [Internet]. Last Update:May 19, 2019.

10. Pombo G. Trombosis venosa superficial: ¿Son todas iguales? Aspectos terapéuticos. Hematología XII Congreso del Grupo CAHT. 2016;20:37-43.

11. Shah NA, Gryskiewicz J, Crutchfield CE. 3rd Successful treatment of a recalcitrant Mondor's cord following breast augmentation. J Clin Aesthet Dermatol. 2014;7:50-1.

12. Hokama A, Fujita J. Mondor disease: an unusual cause of chest pain. Southern Medical Journal 2010;103:1189. 